American Journal of Pharmaceutical Education 2020; 84 (7) Article 7669.

\title{
RESEARCH
}

\section{An Antimicrobial Stewardship Curriculum to Incorporate in the South African Bachelor of Pharmacy Degree Program}

\author{
Yasmine Khan, MPharm, ${ }^{a}$ Lia Kritiotis, $\mathrm{PhD},{ }^{\mathrm{a}}$ Renier Coetzee, PharmD, ${ }^{\mathrm{b}}$ Jane McCartney, PhD, \\ Shirley-Anne Boschmans, $\mathrm{PhD}^{\mathrm{c}}$ \\ ${ }^{a}$ Nelson Mandela University, Port Elizabeth, Eastern Cape, South Africa \\ ${ }^{\mathrm{b}}$ University of Western Cape, Bellville, Western Cape, South Africa \\ ${ }^{c}$ Pharmacy Education Consultant, Johannesburg, Gauteng, South Africa \\ Submitted April 26, 2019; accepted October 8, 2019; published July 2020.
}

Objective. To develop an antimicrobial stewardship curriculum that is suitable for incorporation in the undergraduate pharmacy degree programs offered by South African schools of pharmacy.

Methods. A mixed methods approach was employed involving four consecutive study phases: content analysis, academic review, expert consultation, and curriculum development. The curriculum development phase involved collation of the findings of the prior three study phases.

Results. The final proposed antimicrobial stewardship curriculum included recommendations for: level of incorporation of the curriculum in the undergraduate degree program, minimum contact hours, pedagogical techniques, and assessment methods. The curriculum content was split into four units: pharmacology for antimicrobial stewardship, microbiology for antimicrobial stewardship, antimicrobial stewardship in practice, and hospital antimicrobial stewardship.

Conclusion. An antimicrobial stewardship curriculum that highlights key findings in relation to the role of the pharmacist in antimicrobial stewardship was developed. This was the first such study conducted in South Africa.

Keywords: antimicrobial stewardship, pharmacy, education, curriculum development

\section{INTRODUCTION}

Antimicrobial resistance has been recognized as a threat to global health and safety. ${ }^{1}$ South Africa was identified as one of the top five countries that contributed to the global increase in antibiotic consumption from 2000 to $2010 .^{2}$ In 2018, South African national surveillance data indicated that the total human consumption of antibiotics in South Africa exceeded that of most other countries in the world, suggesting that antimicrobial resistance remains a significant healthcare burden in South Africa. ${ }^{3}$ Antimicrobial stewardship is a healthcare strategy which aims to minimize the progression of antimicrobial resistance by ensuring judicious use of antimicrobial agents. ${ }^{4}$ Pharmacists form part of the "nucleus" of multidisciplinary teams on antimicrobial stewardship. ${ }^{5}$

The World Health Organisation and the South African National Department of Health recognize education of healthcare professionals as an effective strategy for containment of antimicrobial resistance. ${ }^{1,6}$ The South African Society of Clinical Pharmacists noted that the

Corresponding Author: Yasmine Khan, Nelson Mandela University, Port Elizabeth, Eastern Cape, 6019, South Africa. Tel: +2773 (562) 6458. Email: khanyasmine07@gmail.com. training of pharmacists in antimicrobial stewardship was neither formalized or legislated in South Africa, which creates challenges when defining the role of pharmacists in antimicrobial stewardship because the functions of pharmacists in this area would depend upon the amount of training they had received. ${ }^{7}$ The South African National Department of Health has recommended inclusion of antimicrobial stewardship in undergraduate training of pharmacy students as part of the national action plan for containment of antimicrobial resistance in South Africa. ${ }^{8}$ Education of pharmacists on antimicrobial stewardship should be seen as a priority as improved surveillance and intervention by pharmacists has the potential to enhance the quality of antimicrobial prescribing practices. ${ }^{9}$

The undergraduate pharmacy degree program in South Africa, termed the Bachelor of Pharmacy (BPharm) degree, is presented as a four-year undergraduate program. Students graduating from high school (secondary education level) can directly enter a BPharm degree program. Completing the BPharm degree and then an internship year working in selected sectors of pharmacy practice (retail pharmacy, public hospital pharmacy, private hospital pharmacy, pharmaceutical industry or academia) qualifies the graduate to register as 


\section{American Journal of Pharmaceutical Education 2020; 84 (7) Article 7669.}

a generalist pharmacist with the South African Pharmacy Council.

Upon completion of the pharmacy internship, pharmacists are required to complete one year's placement as a community service pharmacist with restricted site of practice. To ensure that all graduates complete a year of community service, the site of practice for the first year of registration is stipulated by the South African Department of Health and is normally in a public sector rural hospital or clinic. On completion of the year of community service, the restriction on site of practice is removed and generalist pharmacists are equipped and permitted to work in any sector of pharmacy practice.

The primary aim of this study was to develop an antimicrobial stewardship curriculum that would be suitable for implementation in Bachelor of Pharmacy degree programs offered by South African schools of pharmacy. The purpose of the curriculum was to ensure that pharmacy students throughout South Africa are equipped with the knowledge and skills required to contribute to antimicrobial stewardship upon entering the practice of pharmacy as newly qualified pharmacists. This study investigated the development of a curriculum for antimicrobial stewardship training at the undergraduate level for a healthcare degree and was the first study of its kind in South Africa.

\section{METHODS}

The final proposed antimicrobial stewardship curriculum was developed based on the results of a crosssectional study that consisted of four consecutive study phases, namely: content analysis, academic review, expert consultation, and curriculum development (Appendix 1). A mixed methods approach was used, with employment of quantitative techniques for the content analysis and academic review phases, and qualitative techniques for the expert consultation phase. Each study phase informed the development of the final proposed antimicrobial stewardship curriculum.

The content analysis phase involved a review of international literature pertaining to antimicrobial stewardship curricula for undergraduate pharmacy students, as well as South African national policy documents, which govern the implementation of antimicrobial stewardship in South Africa. Results from the content analysis allowed for determination of the structure and content of antimicrobial stewardship curricula, which had been successfully developed and implemented internationally, in order to guide the development of the proposed South African curriculum. ${ }^{10-15}$ Furthermore, a review of the national policy documents for antimicrobial stewardship in South Africa enhanced understanding of the principles and strategies that have been recommended by the South African National Department of Health for implementation of antimicrobial stewardship within the context of the South African healthcare system. $., 8,16$

The academic review phase involved the dissemination of questionnaires, which investigated the extent of antimicrobial stewardship teaching in current undergraduate pharmacy degree programs offered by the nine South African schools of pharmacy. Understanding existing practices with regard to antimicrobial stewardship education provided insight into: the variation in the extent and level of antimicrobial stewardship teaching between schools of pharmacy in South Africa, and enabled identification of key principles and strategies that were already emphasized by schools of pharmacy in South Africa when teaching antimicrobial stewardship to undergraduate pharmacy students. Furthermore, the participating academic pharmacists from each school of pharmacy (nominated for participation by the heads of the schools of pharmacy based on their expertise in antimicrobial stewardship education) provided recommendations pertaining to the content that they perceived to be important for inclusion in the proposed curriculum. The academic review phase informed the structure of the final proposed curriculum as it furnished insight into the existing Bachelor of Pharmacy curriculum structures and how antimicrobial stewardship is incorporated into existing programs.

Finally, the expert consultation phase involved qualitative semi-structured interviews by telephone with key role players in antimicrobial stewardship in South Africa. Because antimicrobial stewardship is a multidisciplinary practice, the expert consultation included participants from several health disciplines, including infectious disease specialists $(n=3)$, clinical microbiologists $(n=2)$, an infection prevention control specialist $(\mathrm{n}=1)$, a representative of the Ministerial Advisory Committee for Antimicrobial Resistance in South Africa $(n=1)$, and hospital pharmacists $(n=5)$. Of the hospital pharmacist participants, two were working in public sector, government-funded hospitals and three were employed at different privately funded hospital groups in South Africa.

Public government-funded hospitals in South Africa are generally more resource restricted when compared to private hospitals and rely on compliance to set government standard treatment guidelines. Private hospitals in South Africa are not restricted by government formularies or standard treatment guidelines, thus the approach to antibiotic prescribing and antimicrobial stewardship within public and private sectors may be different. Private and public healthcare facilities also differ in terms of cost 


\section{American Journal of Pharmaceutical Education 2020; 84 (7) Article 7669.}

to the patient. The cost to a patient accessing a private hospital is higher than that of public healthcare facilities, as public healthcare services are largely subsidized by the government.

The interviews explored the perceptions of participants related to: the role of the pharmacist in antimicrobial stewardship in South Africa; the current level of antimicrobial stewardship knowledge and skills possessed by South African pharmacists; and the recommended minimum content for inclusion in an undergraduate antimicrobial stewardship curriculum for pharmacy students. Understanding the perceived role of the pharmacist in antimicrobial stewardship in South Africa, along with the recommended knowledge and skills that pharmacists should possess for effective implementation of antimicrobial stewardship in practice, allowed for the content of the final proposed antimicrobial stewardship curriculum to be practically relevant and applicable to the South African context.

The results of all three study phases were collated in order to develop the final proposed antimicrobial stewardship curriculum for the Bachelor of Pharmacy program. Confidentiality was maintained during the academic review and expert consultation phases as data were not linked to participants or institutional identifiers in order to ensure anonymity in accordance with the Declaration of Helsinki. ${ }^{17}$ Ethical approval to conduct the study was obtained from the Nelson Mandela University Research Ethics Committee. Permission to conduct the academic review with nominated academic pharmacists was granted by the heads of schools from the South African schools of pharmacy. Participants in the expert consultation were required to complete an informed consent declaration prior to conducting the semi-structured interviews by telephone.

\section{RESULTS}

The final proposed antimicrobial stewardship curriculum (Appendix 2) provides minimum recommended antimicrobial stewardship content for inclusion in the Bachelor of Pharmacy degree program offered by South African schools of pharmacy. The proposed structure for the curriculum includes recommendations pertaining to pedagogies, assessment techniques, dedicated contact hours, and year level of inclusion in the program. The proposed content for inclusion in the antimicrobial stewardship curriculum includes key antimicrobial stewardship principles and strategies, which should be incorporated in the undergraduate training of pharmacy students.

We recommend that the proposed antimicrobial stewardship curriculum be incorporated into the third or fourth year of the BPharm program. This recommendation is based on the results of the content analysis and academic review phase. Only a few of the resources which were reviewed in the content analysis provided recommendations on the appropriate year-level for inclusion of antimicrobial stewardship in undergraduate pharmacy education $(9.8 \%, \mathrm{n}=51)$. Literature from the United States recommended incorporation of antimicrobial stewardship training in either the second or third year of the three-year professional component of the Doctor of Pharmacy (PharmD) program. ${ }^{12-14}$ Whereas literature from the United Kingdom recommended inclusion of antimicrobial stewardship training in the third and final year of the fouryear undergraduate pharmacy degree program. ${ }^{11,18}$ Thus, all reviewed literature suggested incorporation of antimicrobial stewardship training in the final two years of the pharmacy degree program.

From the academic review, we found that the content on antimicrobial pharmacology, which provides the foundational knowledge required for understanding of antimicrobial stewardship practices, was presented in the third or fourth year (Table 1) of BPharm programs. Microbiology, which is also an important prerequisite module, was included in all BPharm degree programs either before or concomitantly with antimicrobial pharmacology. Thus, in the context of the South African BPharm program, antimicrobial stewardship training should be incorporated in the third or fourth year to ensure students have completed the prerequisite and foundational content. The exact positioning of the curriculum in the degree program would be informed by the individual requirements and capacity and structure of the BPharm curriculum presented at each school of pharmacy.

Little data were available from the reviewed international literature with regard to the recommended number of contact hours that should be dedicated to antimicrobial stewardship in undergraduate pharmacy training. However, we found that BPharm programs in South Africa dedicated very few contact hours to antimicrobial stewardship, with the majority of the 10 schools

Table 1. Year Level of Presentation of Antimicrobial Pharmacology Content in Bachelor of Pharmacy Degree Programs, as per the Academic Review Questionnaires

\begin{tabular}{lc}
\hline Study Year & $\begin{array}{c}\text { Percentage of Respondents Who Indicated } \\
\text { Inclusion of Antimicrobial Pharmacology }\end{array}$ \\
\hline 1 & 30 \\
2 & 10 \\
3 & 70 \\
4 & 40 \\
\hline
\end{tabular}

a Respondents were permitted to select multiple years of study, ranging from the first year to the final year (fourth year) of study 


\section{American Journal of Pharmaceutical Education 2020; 84 (7) Article 7669.}

of pharmacy surveyed dedicating between one and four contact hours ( $50 \%$ of respondents); or five to nine contact hours ( $40 \%$ respondents) to antimicrobial stewardship. Only one respondent (10\%) indicated that greater than 20 contact hours within their BPharm program were dedicated to antimicrobial stewardship.

Although implementation of the proposed antimicrobial stewardship curriculum encourages an increase in the number of contact hours; the reality of limited time available in current pharmacy programs may only allow for a few hours to be dedicated to antimicrobial stewardship. Thus, a minimum of one to four contact hours was specified in the final proposed antimicrobial stewardship curriculum, but a greater number of contact hours was recommended (from 10 to 19 hours) for the delivery of the proposed antimicrobial stewardship curriculum as the content of the curriculum is extensive. In programs where only limited contact hours are available for dedication to antimicrobial stewardship training, emphasis should be placed on high priority principles and strategies (see curriculum content).

From our content analysis of international literature, we found that most undergraduate pharmacy programs prefer using a mixed methods pedagogy, which involves the combination of multiple pedagogical techniques rather than reliance solely on didactic teaching, for delivery of the antimicrobial stewardship content to pharmacy students. ${ }^{11-13,15,19}$ Additionally, during the interviews we conducted with academic pharmacists, when asked their opinion on the extent to which various antimicrobial stewardship principles should be taught in the curriculum, the majority (on average, 68\%) felt that the principles should be taught in lectures and practical sessions and then applied in practice through work-integrated learning opportunities.

Practical sessions dedicated to antimicrobial stewardship would provide opportunity for students to apply their theoretical knowledge of antimicrobial use in a simulated training session (where students would apply knowledge to provided simulated patient case scenarios). Work-integrated learning opportunities should also provide students with the platform to apply their knowledge and skills in antimicrobial stewardship within the workplace (ideally in the hospital setting). Thus, the final proposed antimicrobial stewardship curriculum (Appendix 2) recommends the utilization of multiple pedagogical techniques, including: didactic lectures, self-study, group activities, class activities, projects, and work-integrated learning placements.

In addition to recommendations for how antimicrobial stewardship principles should be applied in practice, the expert consultation phase with academic pharmacists revealed that work-integrated learning was perceived to be an important tool for teaching antimicrobial stewardship to undergraduate pharmacy students. The importance of work-integrated learning, from the perception of the expert participants, related to the need for pharmacy students to develop skills in application of antimicrobial stewardship knowledge, thus improving the capacity for pharmacy graduates to participate in interprofessional antimicrobial stewardship. Therefore, we strongly recommended that the final proposed antimicrobial stewardship curriculum incorporate opportunities for students to apply their knowledge in the hospital ward environment, and if work-integrated learning is not possible, that students complete case scenarios in which they can apply their knowledge.

As with the pedagogical techniques, the content analysis phase revealed that a mixture of techniques should be used for the assessment of competence following the presentation of antimicrobial stewardship content to pharmacy students. ${ }^{11-14}$ The pedagogical and assessment techniques employed would be determined by the specific institution based upon capacity and preference. However, use of a combination of multiple assessment techniques (eg, written tests/examinations, class activities, case reviews, semester projects, OSCE assessments, pre-class tests, portfolios of clinical activities, essay assignments, and class attendance) is recommended. ${ }^{11-14}$

The content of the final proposed curriculum developed markedly from the content analysis phase to the expert consultation phase. Each study phase allowed for identification of new antimicrobial stewardship principles and strategies, as well as key skills that pharmacy graduates should possess with regards to antimicrobial stewardship prior to entry into practice. The antimicrobial stewardship content has been categorized into four course units: antimicrobial pharmacology, microbiology, antimicrobial stewardship in practice, and hospital antimicrobial stewardship programs.

Because antimicrobial pharmacology and microbiology are prerequisite modules that are presented separately in the BPharm program, unit one and unit two are intended to provide a foundational understanding of the key pharmacological and microbiological principles that underlie antimicrobial stewardship. The content to be included in the proposed antimicrobial stewardship curriculum should, therefore, include only a brief introduction to concepts from pharmacology (management of infectious syndromes, spectra of activity of antimicrobials, and pharmacodynamic and pharmacokinetic concepts affecting antimicrobial dosing) and microbiology (resistance mechanisms, microbiological investigations 


\section{American Journal of Pharmaceutical Education 2020; 84 (7) Article 7669.}

and interpretation of results, and key causative microorganisms for infectious syndromes), as these topics are directly related to the skills required for antimicrobial stewardship.

Unit three provides recommended antimicrobial stewardship principles and strategies that should be incorporated in the undergraduate training of pharmacy students. The content analysis phase highlighted key principles and strategies included in the national policy documents for containment of antimicrobial resistance in South Africa. ${ }^{6,8,16}$ During the academic review phase, academic pharmacists were required to indicate the relative perceived importance of various antimicrobial stewardship principles and strategies for inclusion in the undergraduate training of pharmacy students. The results of the academic review informed the development of relative importance index groupings, which allowed for principles and strategies to be categorized according to their relative importance for inclusion in the proposed antimicrobial stewardship curriculum (Table 2). Furthermore, thematic analysis of the expert consultation interviews enabled determination of perceived importance groupings in order to further categorize principles and strategies according to their perceived importance (Table 3). The results of the academic review phase and expert consultation phase were collated in order to rank antimicrobial stewardship principles and strategies according to relative importance for inclusion in the proposed antimicrobial stewardship curriculum (Appen$\operatorname{dix} 3$ ).

\section{DISCUSSION}

This study aimed to develop a proposed antimicrobial stewardship curriculum that was suitable for implementation in the Bachelor of Pharmacy programs offered by schools of pharmacy in South Africa. The results of the study phases (content analysis, academic review, and expert consultation) informed the development of the final proposed curriculum, thereby fulfilling the aim of the research study.

The content and structure of the proposed curriculum was partially informed by prior studies that involved the development of antimicrobial stewardship curricula for undergraduate pharmacy students, as these studies were included in the review of literature in the content analysis phase. ${ }^{11-15,19}$ However, the structure and content incorporated in the final proposed antimicrobial stewardship curriculum does vary from the recommendations made in other studies because the structure of the final proposed curriculum was aligned with the Bachelor of Pharmacy program in South African schools of pharmacy (academic review phase) as well as the health environment in South Africa. ${ }^{3,6,8,16}$ The number of dedicated contact hours and the level of inclusion in the program was largely informed by consideration of pre-existing pharmacy programs in South Africa. The recommendation for inclusion of

Table 2. Relative Importance Indices for Principles Recommended for Inclusion in an Antimicrobial Stewardship Curriculum for Bachelor of Pharmacy Students $(n=10)$

\begin{tabular}{lllc}
\hline Rank & \multicolumn{1}{c}{ Principle } & RII & RII Group $^{\mathbf{a}}$ \\
\hline 1 & Duration of therapy & 0.950 & 1 \\
1 & Dose optimization & 0.950 & 1 \\
1 & Management of clinical syndromes & 0.950 & 1 \\
4 & Spectrum duplication & 0.925 & 1 \\
4 & Therapeutic drug monitoring & 0.925 & 1 \\
6 & Spectrum streamlining & 0.900 & 1 \\
7 & IV-to-oral switch & 0.875 & 2 \\
7 & Duration of surgical prophylaxis & 0.875 & 2 \\
7 & Antimicrobial stewardship teams & 0.875 & 2 \\
7 & Prospective antimicrobial stewardship strategies & 2 \\
7 & Retrospective antimicrobial stewardship strategies & 0.875 & 2 \\
12 & IV hang time compliance & 0.875 & 2 \\
13 & Strategic objectives and enablers & 0.850 & 2 \\
14 & Antimicrobial cycling & 0.825 & 2 \\
14 & Batching of IV antimicrobials & 0.800 & 2 \\
16 & Culture before commencement & 0.800 & 2 \\
17 & Microscopy review & 0.775 & 2 \\
\hline
\end{tabular}

${ }^{a}$ The intervals for the RII groups were: 0.876 to 1.000 group $1 ; 0.501$ to 0.875 group 2 


\section{American Journal of Pharmaceutical Education 2020; 84 (7) Article 7669.}

Table 3. Perceived Relative Importance of Topics Suggested for Inclusion in an Antimicrobial Stewardship Curriculum as per an Academic Review and Expert Consultations

\begin{tabular}{lll}
\hline Topic & $\begin{array}{c}\text { Perceived Importance in } \\
\text { Expert Consultation }\end{array}$ & $\begin{array}{c}\text { Relative Importance in } \\
\text { Academic Review Phase }\end{array}$ \\
\hline Choice of Agent & High & - \\
Standard Treatment Guideline Compliance & Moderate \\
Microscopy Review & Moderate & Low \\
Spectrum Streamlining & High & High \\
IV-to-Oral Switch & Moderate & Moderate \\
Dose Optimization & High & High \\
Patient Individualization & Low & - \\
Special Population Groups & Low & - \\
Therapeutic Drug Monitoring & Low & High \\
IV Hang-Time Compliance & Low & Low \\
Duration of Therapy & High & High \\
Spectrum Duplication & Moderate & High \\
Surgical Prophylaxis & Moderate & Moderate \\
National Policy & Low & Moderate \\
Diagnostic Stewardship & Moderate & Moderate \\
Infection Prevention and Control & Moderate & - \\
Surveillance & High & - \\
Education & High & - \\
Research & - & - \\
Restrictive Strategies & High & - \\
Prospective and Retrospective Strategies & High & Low \\
Antimicrobial Prescribing Chart - Documenting Indication & Low & - \\
Quality Improvement Interventions & Moderate & - \\
Antimicrobial Stewardship Teams - Antimicrobial Stewardship & High & Moderate \\
Ward Rounds & & Low \\
Institutional Antimicrobial Stewardship Strategies & Low & \\
Batching of Intravenous Antimicrobials & Low & \\
Antimicrobial Cycling & & Low \\
\hline
\end{tabular}

multiple pedagogical techniques and employment of multiple assessment methods is in line with recommendations made in prior studies on antimicrobial stewardship curriculum development. ${ }^{11-13,15,19}$ However, the emphasis placed on work-integrated learning was a unique finding of this study and was based on the recommendations made by antimicrobial stewardship experts in South Africa during the expert consultation phase.

The content of the final proposed antimicrobial stewardship curriculum was largely informed by national policy documents, which govern the implementation of antimicrobial stewardship in South Africa. Although similar antimicrobial stewardship principles have been recommended in prior studies for inclusion in undergraduate pharmacy antimicrobial stewardship training, contextualization of the content to the South African national recommendations introduced variation in the final content proposed in this study. Furthermore, ranking the relative importance of various antimicrobial stewardship principles and strategies for inclusion in the final proposed curriculum was a unique finding of this study and considered the recommendations made by both academic pharmacists and a multi-disciplinary panel of antimicrobial stewardship experts in South Africa.

Even though the study considered multiple sources of data to inform the development of the final proposed antimicrobial stewardship curriculum for South African undergraduate pharmacy degree programs, the study is not without limitations. Because the content analysis only considered online published resources pertaining to international antimicrobial stewardship curricula, there was no consideration of the structure and content of additional antimicrobial stewardship curricula that have not been published. Thus, the conclusions drawn from the review of published international antimicrobial stewardship curricula may not be representative of the antimicrobial 


\section{American Journal of Pharmaceutical Education 2020; 84 (7) Article 7669.}

stewardship curricula that have been developed and implemented internationally. The academic review included the responses and perceptions of only one nominated participant from each school of pharmacy in South Africa. Thus, the perceptions and recommendations of all academic pharmacists in South Africa were not considered. The sampling technique used in the academic review and expert consultation phase relied upon purposive sampling instead of random sampling and thus had the potential to introduce bias.

The results of the study may form the foundation for further potential research into antimicrobial stewardship education in undergraduate health degree programs in South Africa and the role of the pharmacist in antimicrobial stewardship practice in South Africa. The feasibility for implementation of the proposed antimicrobial stewardship curriculum should be further investigated. The impact of the introduction of the proposed antimicrobial stewardship curriculum on the antimicrobial stewardship knowledge and skills possessed by pharmacy students before-and-after implementation of the curriculum should also be investigated.

\section{CONCLUSION}

The study described the development of a proposed antimicrobial stewardship curriculum and highlighted key findings in relation to the role of the pharmacist in antimicrobial stewardship in South Africa. This is the first study conducted in South Africa that involved the development of an undergraduate antimicrobial stewardship curriculum for pharmacy students.

The inclusion of multiple sources of data (literature, academic pharmacists, and a multi-disciplinary panel of antimicrobial stewardship experts in South Africa) in the development of the final proposed antimicrobial stewardship curriculum allowed for the incorporation of various perceptions, which were derived from unique perspectives. South African antimicrobial stewardship policy guidelines and the perceptions of South African healthcare professionals informed the content of the final proposed antimicrobial stewardship curriculum, which is contextualized to the South African healthcare setting. However, the methodology employed in this study could serve as a model for the development of similar antimicrobial stewardship curricula for inclusion in other undergraduate healthcare degrees (medical, nursing, dental, veterinarian, etc) nationally and internationally.

\section{ACKNOWLEDGMENTS}

The authors acknowledge the contributions made by Dr Danie Venter for his statistical assistance and Dr Timothy Pittaway for his guidance in qualitative data analysis. Furthermore, the authors thank the healthcare professionals who participated in the study and provided their insights, which informed the development of this curriculum. The views expressed in this article reflect those of the authors and do not necessarily reflect recommendations by the Nelson Mandela University or University of the Western Cape. The research was supported by the Postgraduate Research Scholarship from Nelson Mandela University as well as the DormehlCunningham Trust.

\section{REFERENCES}

1. World Health Organisation. WHO global strategy for containment of antimicrobial resistance. http://www.who.int/drugresistance/ WHO_Global_Strategy.htm/en/. Accessed July 7, 2020.

2. Van Boeckel T, Gandra S, Ashok A, et al. Global antibiotic consumption 2000 to 2010: an analysis of national pharmaceutical sales data. The Lancet Infect Dis. 2018;14(8):742-750.

3. National Department of Health, South Africa. Surveillance for antimicrobial resistance and consumption of antibiotics in South

Africa. http://www.health.gov.za/index.php/component/ phocadownload/category/199-antimicrobial-resistance\#. Accessed July 7, 2020.

4. Duguid M, Cruickshank M. Antimicrobial stewardship in Australian hospitals. https:/www.safetyandquality.gov.au/wpcontent/uploads/2011/01/Antimicrobial-stewardship-in-AustralianHospitals-2011.pdf. Accessed July 7, 2020.

5. Mendelson M. Role of antibiotic stewardship in extending the age of modern medicine. S Afr Med J. 2015;105(5):414-419.

6. National Department of Health, South Africa. Antimicrobial resistance national strategy framework 2014-2024. http:// www.mm3admin.co.za/documents/docmanager/3C53E82B-24F249E1-B997-5A35803BE10A/00090160.pdf. Accessed July 7, 2020.

7. Schellack N, Bronkhorst E, Coetzee R, et al. SASOCP position statement on the pharmacist's role in antibiotic stewardship. $S$ Afr J Infect Dis. 2018;33(1):28-35.

8. National Department of Health, South Africa. South African antimicrobial resistance national strategy framework: a one health approach 2018-2024. http://www.health.gov.za/index.php/ component/phocadownload/category/199-antimicrobial-resistance\#. Accessed July 7, 2020

9. MacDougall C, Polk R. Antimicrobial stewardship programs in health care systems. Clin Microbiol Rev. 2005;18(4):638-656.

10. Barlam T, Cosgrove S, Abbo L, et al. Implementing an antibiotic stewardship program: guidelines by the Infectious Diseases Society of America and the Society for Healthcare Epidemiology of America. Clin Infect Dis. 2016;62(10):51-77.

11. Castro-Sanchez E, Drumright L, Gharbi M, Farrell S, Holmes A. Mapping antimicrobial stewardship in undergraduate medical, dental, pharmacy, nursing and veterinary education in the UK. PLoS One. 2016;11(2): 0150056.

12. Falcione B, Meyer S. Instructional design and assessment: development of an antimicrobial stewardship-based infectious disease elective that incorporates human patient simulation technology. Am J Pharm Educ. 2014;78(8):1-8. N 13. Gauthier T, Sherman E, Unger N. Instructional design and assessment: an elective course on antimicrobial stewardship. Am J Pharm Educ. 2015;79(10):1-7. 


\section{American Journal of Pharmaceutical Education 2020; 84 (7) Article 7669.}

14. Mathias C. A learner-led, discussion-based elective on emerging infectious disease. Am J Pharm Educ.

2015;79(6):1-10.

15. Snedden J, Gilchrist M, Wickens H. Development of an expert professional curriculum for antimicrobial pharmacists in the UK. $J$ Antimicrob Chemother. 2015;70(5):1277-1280.

16. National Department of Health, South Africa. Guidelines on implementation of the antimicrobial strategy in South Africa: One health approach \& governance. http://nahf.co.za/guidelines-on-the- implementation-of-the-antimicrobial-strategy-in-south-africa-201707-14/. Accessed July 7, 2020.

17. World Medical Association. World Medical Association Declaration of Helsinki. JAMA. 2013;310(20): 2191-2194.

18. Pulcini C, Gyssens I. How to educate prescribers in antimicrobial stewardship practices. Virulence. 2013;4(2):192-202.

19. Infectious Diseases Society of America. Combatting antimicrobial resistance: policy recommendations to save lives. Clin Infect Dis. 2011;52(5):397-428. 


\section{American Journal of Pharmaceutical Education 2020; 84 (7) Article 7669.}

Appendix 1. Summary of the Methodology Employed for the Development of the Proposed South African Antimicrobial Stewardship Curriculum for the Bachelor of Pharmacy Program

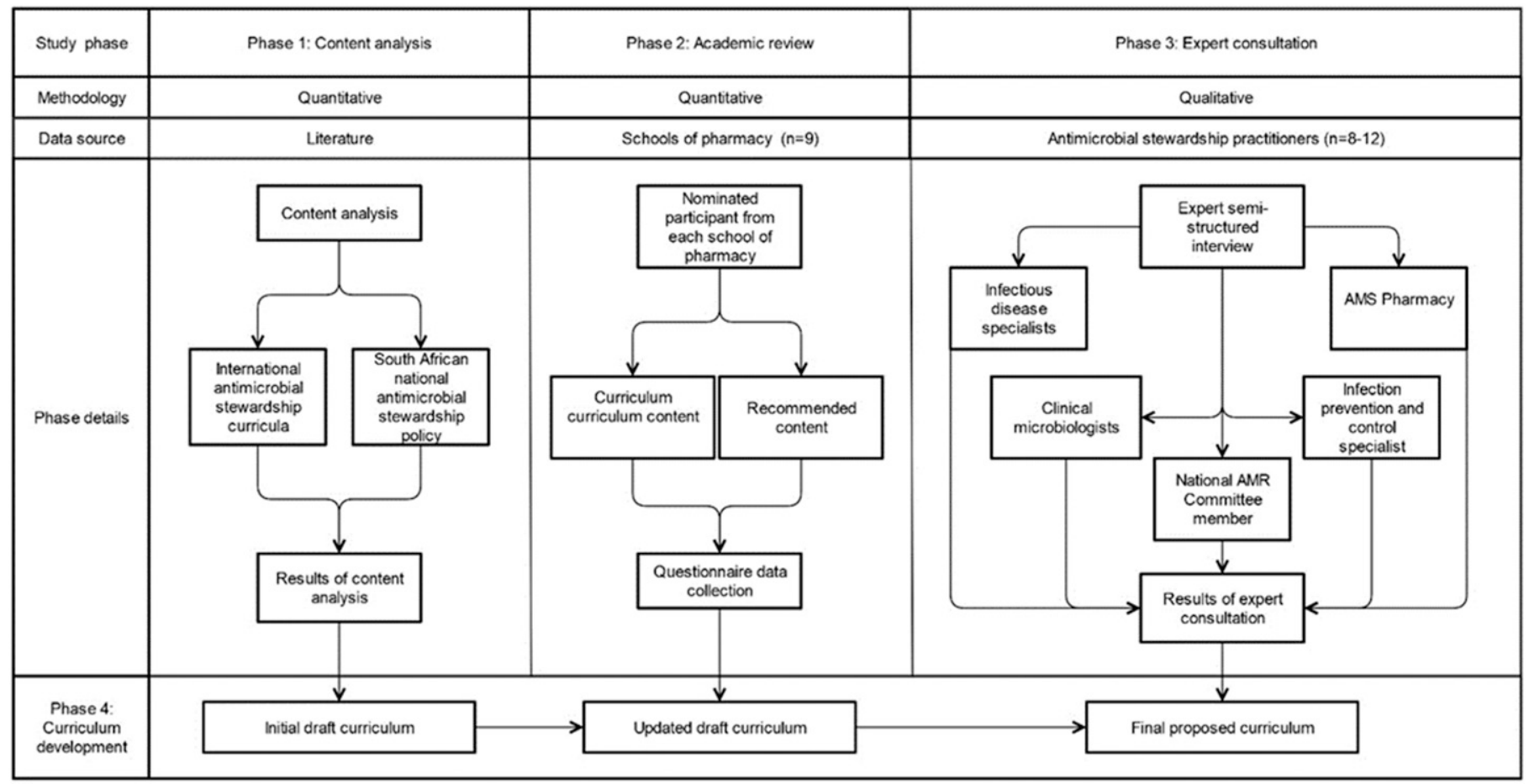

The study methodology involved four consecutive phases: a content analysis; academic review; expert consultation; and curriculum development. The content analysis phase included an in-depth review of literature pertaining to international antimicrobial stewardship curriculum, and antimicrobial stewardship in South Africa. The academic review phase was conducted to determine the extent to which antimicrobial stewardship is currently included in undergraduate pharmacy programs in South African schools of pharmacy. The expert consultation phase established input from actively practicing antimicrobial stewardship professionals on the roles of and knowledge required by newly qualified pharmacists to function effectively in antimicrobial stewardship. The collation of the findings from the first three phases of the study occurred during the fourth and final study phase: the curriculum development phase. The curriculum development phase ran concurrently with the first three study phases, with the curriculum being developed throughout the study as a result of the incorporation of findings from the various study phases. 


\section{American Journal of Pharmaceutical Education 2020; 84 (7) Article 7669.}

Appendix 2. Final Proposed Curriculum: Antimicrobial Stewardship for Pharmacy Students

Course units:

Four course units have been developed for the purposes of educating pharmacy students on antimicrobial stewardship.

Antimicrobial pharmacology and microbiology are discussed in two course units, in order to revise the key pharmacological and microbiological principles which influence the practice of antimicrobial stewardship. The remaining two course units contain content on Antimicrobial stewardship in practice, and hospital antimicrobial stewardship programs, respectively.

\section{Course pre-requisites:}

Understanding the principles of antimicrobial pharmacology and microbiology is a requirement for understanding and accurate application of antimicrobial stewardship principles. Thus, prior to introduction to the antimicrobial stewardship curriculum, students should have successfully completed module/s pertaining to antimicrobial pharmacology, and microbiology for pharmacy students, as determined by the institution's curriculum.

\section{Course structure:}

Year level of incorporation into the BPharm program:

The course units are intended to be incorporated into the degree program as a single course, in the third or fourth years of the pharmacy degree program and should be incorporated such that students have met pre-requisite content. The exact level of inclusion of the antimicrobial stewardship curriculum (third- or fourth-year level) would be determined by the institution, based upon the curriculum capacity within each respective year, and at which level in the program pre-requisite module/s would be adequately delivered.

\section{Minimum and recommended contact hours:}

It is recommended that a minimum of 1-4 contact hours be dedicated for implementation of the antimicrobial stewardship curriculum. It is highly recommended that a greater number of contact hours be dedicated to antimicrobial stewardship where possible, with a recommended range of 10-19 hours for optimal delivery of the final proposed antimicrobial stewardship curriculum. Where an increased number of contact hours can be allocated (based upon availability within the pre-existing BPharm curriculum), additional time should be spent on the recommended antimicrobial stewardship principles, in proportion to their relative importance groupings.

\section{Pedagogical recommendations:}

The intended learning outcomes for each course unit and potential pedagogical foci which could be employed for the purposes of achieving the intended learning outcomes, has been outlined below (see intended learning outcomes). The specific pedagogies employed should be chosen at the discretion of the persons responsible for implementation of the curriculum, however, it is recommended that a mixture of pedagogical techniques is employed for the delivery of content on antimicrobial stewardship. Application of antimicrobial stewardship knowledge is strongly encouraged and should be achieved through incorporation of case scenarios within didactic/simulated practical sessions and /or allowing opportunity for application within a work-integrated learning placement.

Work-integrated learning opportunities should allow for pharmacy students to apply the antimicrobial stewardship principles gained through didactic sessions to patient cases, as encountered within the various sectors of pharmacy practice. Ideally, application of antimicrobial stewardship principles should be ensured within multiple practice settings, including the hospital sector (private and public), retail/community pharmacy and primary healthcare clinics.

Where possible, work-integrated learning placements should allow for pharmacy students to participate in an antimicrobial stewardship ward round, where the interactions of the multidisciplinary antimicrobial stewardship team can be observed. Active participation in discussions around antimicrobial stewardship during work-integrated learning should be encouraged, and where possible, assessed. Participation in antimicrobial stewardship discussions is crucial for the development of skills in interprofessional interaction by pharmacy students (a skill which was identified as deficient in current pharmacy students and practicing pharmacists by antimicrobial stewardship experts in South Africa). Where application through work-integrated learning is not possible, case scenarios should be incorporated into the didactic sessions, reflecting patient cases which students are likely to encounter in various pharmacy practice settings. Opportunities to practice the processes of antimicrobial stewardship interventions should be provided, whether in work-integrated placements or practical sessions. 


\section{American Journal of Pharmaceutical Education 2020; 84 (7) Article 7669.}

(Continued)

Assessment of competency:

Potential assessment techniques which could be employed to ensure competence in the antimicrobial stewardship course include: written test/examination, class activities, case reviews, semester projects, OSCE assessments, pre-class tests, development of a portfolio of clinical activities, essay assignments and class attendance. A combination of two or more methods of assessment is encouraged, to ensure application of knowledge in multiple settings.

\section{Intended learning outcomes:}

Unit 1: Antimicrobial pharmacology for stewardship

This unit relates to the pharmacological principles which are essential for implementation of antimicrobial stewardship practices. At the end of this unit, the student should be able to:

- Apply knowledge of antimicrobial pharmacology to the management of common infectious disease syndromes.

- Establish a knowledge of the spectrum of activity of antimicrobial agents.

- Understand the importance of pharmacokinetic and pharmacodynamics principles in the determination of antimicrobial dosing, in terms of dosage and frequency.

Potential instructional foci: didactic lectures, self-study/review, group activities, class activities/tutorials

Unit 2: Microbiology for stewardship

This unit relates to the microbiological principles which contribute to antimicrobial stewardship practices. At the end of this unit, the student should be able to:

- Understand the basic processes involved in the development of resistance by microorganisms.

- Identify the key microbiological processes involved in the antimicrobial stewardship review.

- Interpret and communicate the clinical implications of microbiological results on antimicrobial therapy.

- Establish knowledge of the key causative microorganisms in common infectious syndromes (link to the spectrum of activity covered in unit 1).

Potential instructional foci: didactic lectures, guest lecturer (clinical microbiologists) self-study/review, group activities, class activities/tutorials

Unit 3: Antimicrobial stewardship in practice

This unit relates to the key principles and strategies involved in antimicrobial stewardship implementation in practice. At the end of this unit, the student should be able to:

- Understand the basic framework for the South African antimicrobial resistance program, and the placement of antimicrobial stewardship within the program.

- Identify and apply the key antimicrobial stewardship strategies utilised for achievement of antimicrobial stewardship in practice.

- Understand the antimicrobial prescribing principles underlying antimicrobial stewardship review and apply the principles to the review of antimicrobial prescribing for appropriateness.

A list of antimicrobial stewardship strategies and antimicrobial prescribing principles has been determined for inclusion in unit 3. The strategies and principles have been grouped according to relative importance (Appendix 3), which should determine the proportion of contact time dedicated to each principle (dependant on the available total contact time within pre-existing curricula). Principles which have been categorised within a higher relative importance group should be allotted more contact time and focus than principles which have been categorised in a lower relative importance group.

Unit 4: Hospital antimicrobial stewardship

This unit relates to the implementation of antimicrobial stewardship programs in the hospital environment. At the end of this unit, the student should be able to:

- Develop and evaluate tools/plans for the effective implementation, monitoring and enhancement of antimicrobial stewardship in practice.

- Apply the concepts of antimicrobial stewardship to the hospital setting, both for the review of infectious disease cases and for the surveillance of antimicrobial stewardship implementation.

Potential instructional foci: Class activities, projects, work-integrated learning (hospital setting) 


\section{American Journal of Pharmaceutical Education 2020; 84 (7) Article 7669.}

Appendix 3. Course Map Summarizing the Content Included in the Final Proposed Antimicrobial Stewardship Curriculum for the South African Bachelor of Pharmacy Program

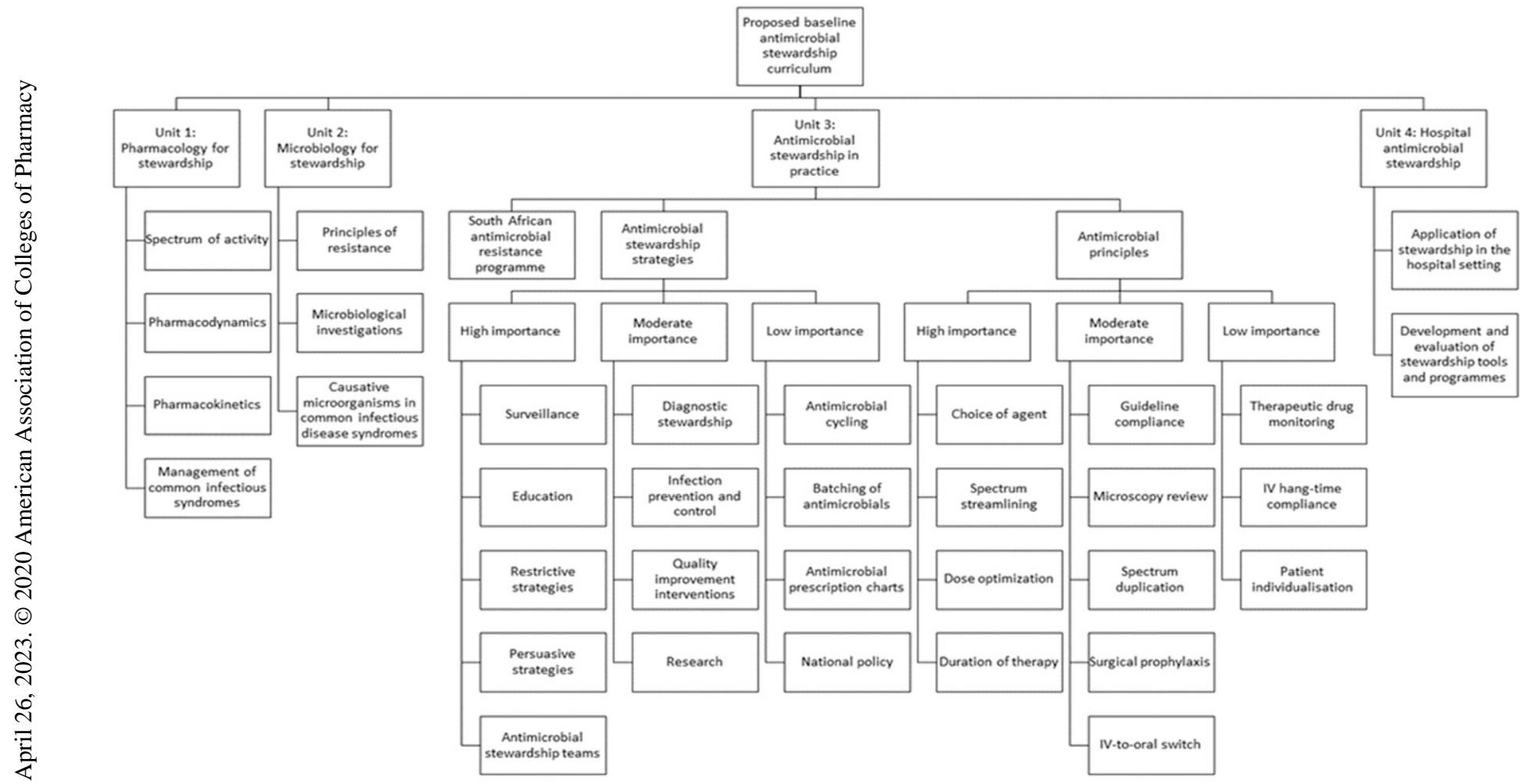

